\title{
Preface
}

\section{PET/CT: Adding Value to \\ Patient Outcomes and Health Care Delivery}

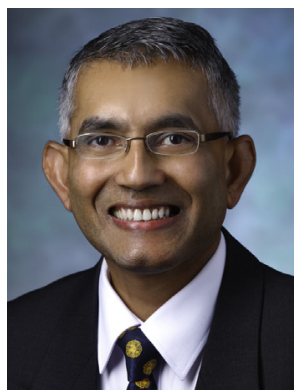

Rathan M. Subramaniam, MD, PhD, MPH Editor

This is the first issue in the two-part PET Clinic series of "PET/CT and Patient Outcomes." Adding value to patients and patient outcomes at reasonable cost, which a society can afford, is the founding principle for providing an affordable health care delivery. Advanced imaging tests, such as CT, PET/ $\mathrm{CT}$, and MRI, are expensive, and their utilization and benefit must be justified for the clinical context, benefit the patients, and reduce the unnecessary waste of resources in providing care. The cost of $\mathrm{PET} / \mathrm{CT}$ is entirely justifiable, if the information provided by PET/CT leads to cost saving by avoiding delays in therapy decision-making and starting therapies, by avoiding very expensive and inappropriate therapies, and by identifying therapy failures early so that appropriate therapy can be commenced sooner, improves patient quality of life, and predicts patient outcomes.

PET/CT provides value to patients by providing additional information to clinicians in assessing the disease stage, deciding on appropriate therapy, assessing the therapy success or failure, predicting patient outcomes, and providing additional information about disease status in followup, if a patient has symptoms or signs suspicious of recurrence. As carefully put together by an international group of authors in this issue, PET/CT provides added value to patients and their outcomes in head and neck cancer, lung cancer, breast cancer, esophageal cancer, lymphoma, multiple myeloma, melanoma, prostate cancer, thyroid cancer, and radiation therapy planning.
Depending on the tumor type, the strength of evidence for the added value and patient outcome varies, in the appropriate clinical context.

It is also important to recognize that PET/CT has pitfalls. Lack of standardization in reporting can lead to harmful effects, such as unnecessary biopsies, and inappropriate utilization, such as surveillance imaging, which result in patient anxieties and drive up the cost of health care delivery. As a professional community, it is our responsibility to mitigate these harmful effects and provide the best care to our patients at the right time, in the right setting, and by the right teamwork. We should be careful and thoughtful before recommending biopsies and additional imaging tests and strongly discourage performing surveillance PET/CT imaging without symptoms or signs suggestive of recurrence in follow-up. Our collaborative teamwork with our clinicians would enhance our service to our patients and reduce many of the harmful effects. This will truly magnify the benefits and added value of PET/CT to all of our patients.

Rathan M. Subramaniam, MD, PhD, MPH Russell H. Morgan Department of Radiology and

Radiology Science Johns Hopkins Medical Institutions 601 North Caroline Street/JHOC 3235 Baltimore, MD 21287, USA rsubram4@jhmi.edu 